\title{
REMOÇÃO DE COMPOSTOS ALCALINOS PRESENTES NA PREPARAÇÃO DE CATALISADORES CONP/SBA-15
}

\author{
G. G. CHER ${ }^{1}$, L. S. da SILVA² e P. A. ARROYO² \\ ${ }^{1}$ Universidade Estadual de Maringá, Departamento de Química \\ ${ }^{2}$ Universidade Estadual de Maringá, Departamento de Engenharia Química \\ E-mail para contato: arroyo@deq.uem.br
}

\begin{abstract}
RESUMO - No presente trabalho foi realizada a síntese de CoNP/SBA-15 visando à Síntese de Fischer-Tropsch. Durante a síntese dos catalisadores houve contaminação das amostras com nitrato de sódio, devido à natureza do agente redutor utilizado na obtenção da fase metálica (CoNPs). A tentativa de remoção dos compostos de sódio foi realizada por meio de um simples tratamento de lixiviação, utilizando etanol e EDTA como solvente e agente complexante, respectivamente, seguido por um tratamento térmico em temperatura adequada. Verificou-se que o tratamento utilizando EDTA é mais efetivo na retirada quase que completa da fase alcalina das amostras. Também foi verificado que a etapa de tratamento térmico foi essencial para alcançar este resultado.
\end{abstract}

\section{INTRODUÇÃO}

O processo de Fischer Tropsch é uma tecnologia eficiente na conversão de fontes de carbono, como, por exemplo, carvão, gás natural e biomassa, em produtos de alto valor agregado e menor poder poluente (Dry, 1996). Deste modo, uma vez que o Brasil apresenta reservas de gás natural abundantes, a síntese de Fischer Tropsch ganha destaque visto que é umas das principais etapas no processo GTL (gas to liquid), o qual transforma gás natural em hidrocarbonetos líquidos (Ramos et al., 2011).

Embora vários metais sejam ativos na Síntese de Fischer-Tropsch (SFT), apenas o ferro e o cobalto são economicamente viáveis para serem utilizados como metal ativo em escala industrial. (O'brien et al., 1997). Catalisadores a base de cobalto têm sido bastante estudados na SFT devido a sua elevada atividade, alta resistência à desativação e baixa atividade na reação de shift (Martínez et al., 2003). Além disso, o cobalto é o metal mais indicado quando o gás de síntese é obtido a partir do gás natural, devido à elevada razão $\mathrm{H}_{2} / \mathrm{CO}$ (Davis, 2003).

A fim de alcançar uma maior quantidade de sítios ativos, catalisadores suportados são comumente utilizados, por permitirem a dispersão da fase metálica em uma elevada área específica. Por possuir estreita distribuição de tamanho, elevada porosidade e elevada estabilidade térmica, em relação a outros materiais mesoporosos (Zhao et al., 2000), a SBA15 se apresenta como uma boa alternativa a ser utilizada como suporte de catalisadores.

Metais alcalinos e alcalinos terrosos podem ser acidentalmente inseridos nos catalisadores destinados à STF durante as etapas de preparação. Estes componentes podem ser introduzidos, por exemplo, por meio de impurezas presentes na água, precursores de 
promotores e metais ativos e, também, a partir de equipamentos utilizados no processo. A literatura mostra que a atividade dos catalisadores diminui e a seletividade a $\mathrm{CO}_{2}$ aumenta, conforme aumenta a quantidade de sódio presente nos catalisadores (Lillebo et al., 2013; Borg et al., 2011; Balonek et al., 2010). Acredita-se que estes comportamentos são em menor parte atribuídos a efeitos geométricos da superfície alcalina e em maior parte a efeitos eletrônicos, os quais podem afetar a adsorção e dessorção das moléculas de $\mathrm{H}_{2}$ e $\mathrm{CO}$ (Balonek et al., 2010). Portanto, há a necessidade de remoção destes compostos alcalinos.

No presente trabalho foi realizado um procedimento de lavagem de catalisadores de CoNP/SBA-15, visando à remoção de compostos de sódio presentes durante o procedimento de síntese utilizado, por meio de um procedimento simples e rápido, permitindo que os catalisadores possam ter um melhor desempenho na STF. Os solventes utilizados nas lavagens foram o etanol e o EDTA. O primeiro foi escolhido por ser um bom solvente para o nitrato de sódio e o segundo por ser um bom agente complexante. A presença da fase alcalina teve como origem a utilização de borohidreto de sódio como agente redutor na síntese da fase ativa (CoNPs).

\section{EXPERIMENTAL}

\subsection{Síntese dos Catalisadores}

A SBA-15 utilizada como suporte do catalisador foi sintetizada conforme o procedimento proposto por Zhao et al. (1998) e Zhao et al. (2000). A síntese das nanopartículas de cobalto (CoNPs) foi realizada de acordo com o método proposto por Zhao et al. (2003).

As CoNPs foram incorporadas aos suportes mesoporosos por impregnação incipiente. O método consistiu em gotejar lentamente a suspensão de nanopartículas sobre o suporte, de forma a se obter teores entre 4 e $8 \%$ em massa de cobalto, misturando com espátula até a formação de um gel. Foram escolhidos tamanhos de nanopartículas entre 2,0 e 7,0 nm para impregnação no suporte. Posteriormente, a amostra foi mantida em um dessecador, a temperatura ambiente, sob vácuo para secagem.

\subsection{Lavagem dos catalisadores}

As amostras impregnadas foram submetidas a um tratamento com o objetivo de remover os compostos de sódio presentes. O tratamento consistiu numa etapa de lavagem e em uma etapa de tratamento térmico. Assim, foram adotados os seguintes procedimentos de lixiviação: as amostras foram lavadas uma vez com etanol 99,5\%, numa proporção volumétrica de 1:3, respectivamente, e, em seguida, centrifugadas por 10 minutos a $3500 \mathrm{rpm}$; as amostras foram lavadas cinco vezes com etanol 99,5\% em uma proporção volumétrica de 1:3 de catalisador:etanol e centrifugadas por 10 minutos a $3500 \mathrm{rpm}$; e as amostras foram lavadas cinco vezes com EDTA $(0,3 \mathrm{~mol} / \mathrm{L})$, em uma proporção volumétrica de 1:3 de catalisador: EDTA, e centrifugadas por 10 minutos a $3500 \mathrm{rpm}$. Após a etapa de centrifugação, as amostras foram colocadas em um dessecador para secagem, por sete dias. Posteriormente, os catalisadores foram submetidos a um tratamento térmico por um período de três horas a 150 e $300^{\circ} \mathrm{C}$. A Tabela 1 apresenta a nomenclatura e condições utilizadas no tratamento, para cada amostra. 
Tabela 1 - Nomenclatura e condições utilizadas no processo de lavagem das amostras.

\begin{tabular}{cccc}
\hline Amostra & Solvente & Quantidade de lavagens & Temperatura do tratamento térmico $\left({ }^{\circ} \mathrm{C}\right)$ \\
\hline 1 & Etanol & 1 & 150 \\
2 & Etanol & 1 & 300 \\
3 & Etanol & 5 & 150 \\
4 & Etanol & 5 & 300 \\
5 & EDTA & 5 & 150 \\
6 & EDTA & 5 & 300 \\
\hline
\end{tabular}

\subsection{Caracterizações}

Difração de Raios X (DRX): as análises de DRX das amostras foram realizadas no Departamento de Engenharia Química (DEQ/UEM), utilizando-se um difratômetro da marca Shimadzu, modelo XRD6000, com um tubo de cobre e filtro de níquel, radiação $\mathrm{Cu}-\mathrm{K} \alpha$ $(1,54 \AA)$, velocidade de varredura de $0,5^{\circ} / \mathrm{min}$ de 5 a $60^{\circ}, 40 \mathrm{kV}$ e $30 \mathrm{~mA}$. As fendas utilizadas foram de $0,5^{\circ}$ (divergência), $0,5^{\circ}$ (espalhamento) e de $0,30 \mathrm{~mm}$ (recepção). Os picos foram identificados por comparação com fichas PCPDFWIN, com o auxílio do pacote computacional PCPDFWIN v. 2.3.

Espectroscopia na região do Infravermelho com Transformada de Fourier (FTIR): a identificação de compostos presentes na superfície de Co/SBA-15 foi realizada por meio de análise por FTIR, usando um espectrofotômetro Bruker Vertex $70 \mathrm{~V}$. Para realização da análise foi necessário a preparação de pastilhas de $\mathrm{KBr}$. Primeiramente, misturaram-se $198 \mathrm{mg}$ de $\mathrm{KBr}$ com $2 \mathrm{mg}$ de cada amostra até a mistura se tornar homogênea. Em seguida, a mistura foi pressurizada até a formação de pastilhas que tivessem um tamanho e espessura adequada para utilização no equipamento.

\section{RESULTADOS E DISCUSSÃO}

Os resultados correspondentes à análise por Difração de Raios $\mathrm{X}$ dos catalisadores são mostrados na Figura 1. Nos difratogramas correspondentes ao catalisador sem tratamento de lavagem e em alguns catalisadores após o tratamento observam-se picos em ${ }^{0} 2 \theta=29,5$ e ${ }^{0} 2 \theta$ $=39$ que são indexados aos planos (104) e (113), respectivamente, indicativos da presença de nitrato de sódio (PCPDFWIN 89-0311). Este composto é um produto secundário na reação de redução do nitrato de cobalto com borohidreto de sódio. A presença destes compostos não é interessante em catalisadores utilizados para a Síntese de Fischer-Tropsch (SFT).

Não foram observados picos correspondentes a espécies de cobalto oxidadas, que seriam observados em ${ }^{0} 2 \theta=36,9$ para o $\mathrm{Co}_{3} \mathrm{O}_{4}$ e em ${ }^{0} 2 \theta=42$ para o $\mathrm{CoO}$, e nem para espécies de cobalto metálicas que teriam um pico localizado em torno de ${ }^{0} 2 \theta=45$, de acordo com as fichas PCPDFWIN 78-1970, 78-0431 e 88-2325, respectivamente. Isto poderia ser justificado pelo fato das CoNPs estarem na faixa limite de detecção do equipamento $(20 \mathrm{~nm})$. Por outro lado, isto mostra que após o tratamento térmico as partículas de cobalto não aglomeraram de forma significativa, o que é vantajoso, já que a manutenção do tamanho das CoNPs impregnadas é essencial para se obter elevada atividade e seletividade a $\mathrm{C}_{5+}$ na Síntese de Fischer-Tropsch (Wang et al., 2012; Yang et al., 2010; Bezemer et al., 2006). 

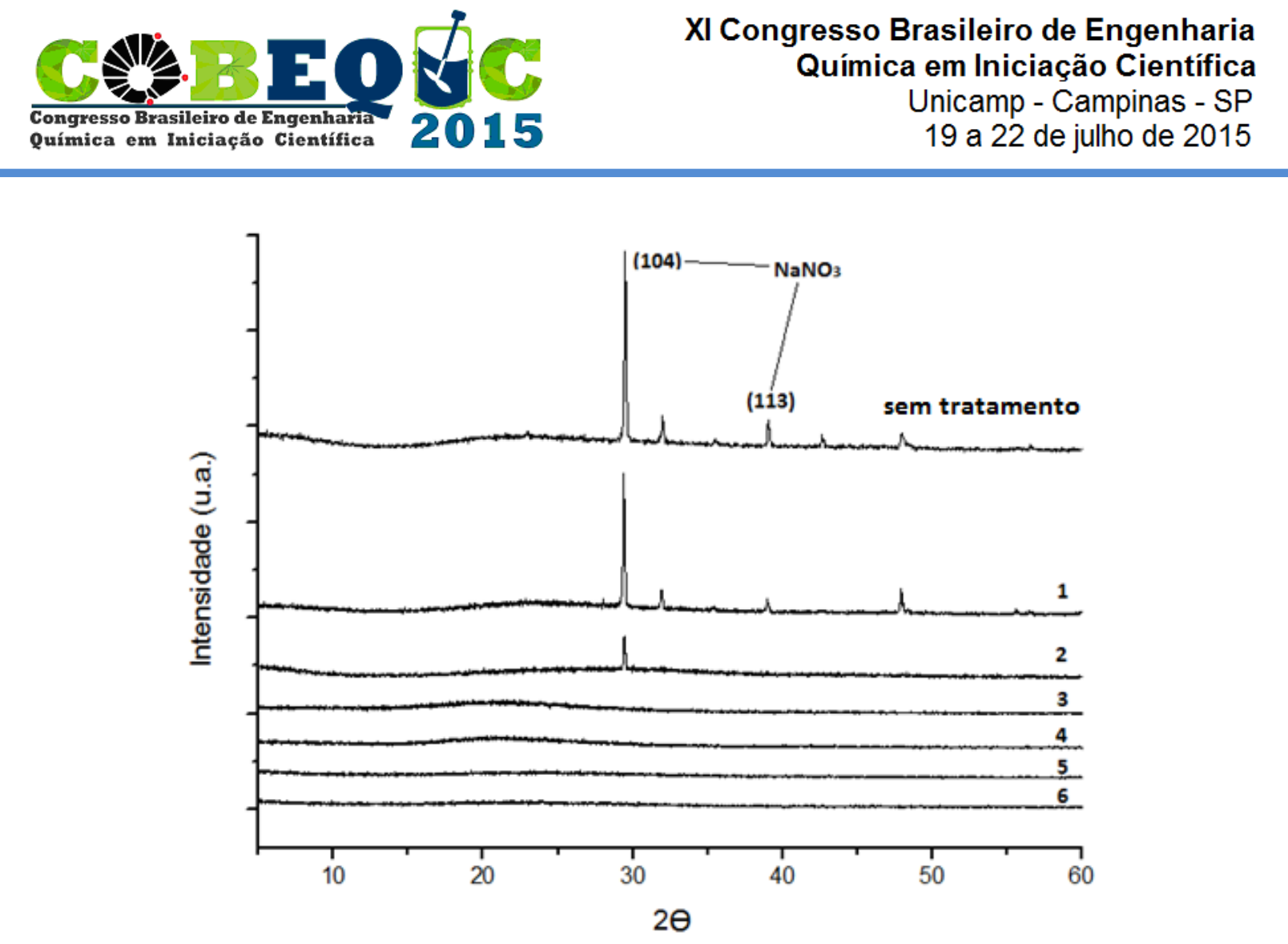

Figura 1 - Difrações de raios $\mathrm{X}$ dos catalisadores antes e após os processos de lavagem.

Observa-se pela Figura 1 que a etapa de lavagem auxilia na remoção dos compostos de sódio e que a etapa de tratamento térmico também exerce influência significativa. Lavando-se os catalisadores com etanol apenas uma vez, a quantidade de nitrato de sódio removido aumenta após tratamento térmico a uma temperatura mais elevada. Isto sugere que parte dos compostos alcalinos, os quais não são removidos com o solvente após a centrifugação, são removidos com o solvente evaporado durante o tratamento térmico. Lavando-se os catalisadores cinco vezes com etanol e com EDTA, não se verifica por DRX a presença da fase indesejada.

Vale ressaltar aqui que a fase alcalina poderia ainda estar presente nos catalisadores, caso as partículas estivessem com tamanhos inferiores a $20 \mathrm{~nm}$. Por esta razão, foi realizada a análise por FTIR, a qual é mostrada na Figura 2, com o objetivo de verificar se ainda havia presença dos compostos de sódio nas amostras.

É possível observar na Figura 2 uma das bandas característica do nitrato de sódio, a qual, de acordo com Tam et al. (1997.), está localizada em $1366 \mathrm{~cm}^{-1}$. Também se observa que após os tratamentos utilizando etanol como solvente ainda há presença de fase alcalina, enquanto que o tratamento utilizando EDTA foi mais eficiente na remoção dos compostos. Neste último caso, o tratamento térmico a uma maior temperatura auxiliou na remoção quase que completa do nitrato de sódio. Desta maneira, é possível verificar que o poder de complexação do EDTA é maior que o poder de dissolução do etanol, possibilitando a remoção dos compostos de sódio após tratamento térmico, em temperatura adequada. Sem a presença da fase alcalina o catalisador pode ter um melhor desempenho na Síntese de FischerTropsch (SFT). 


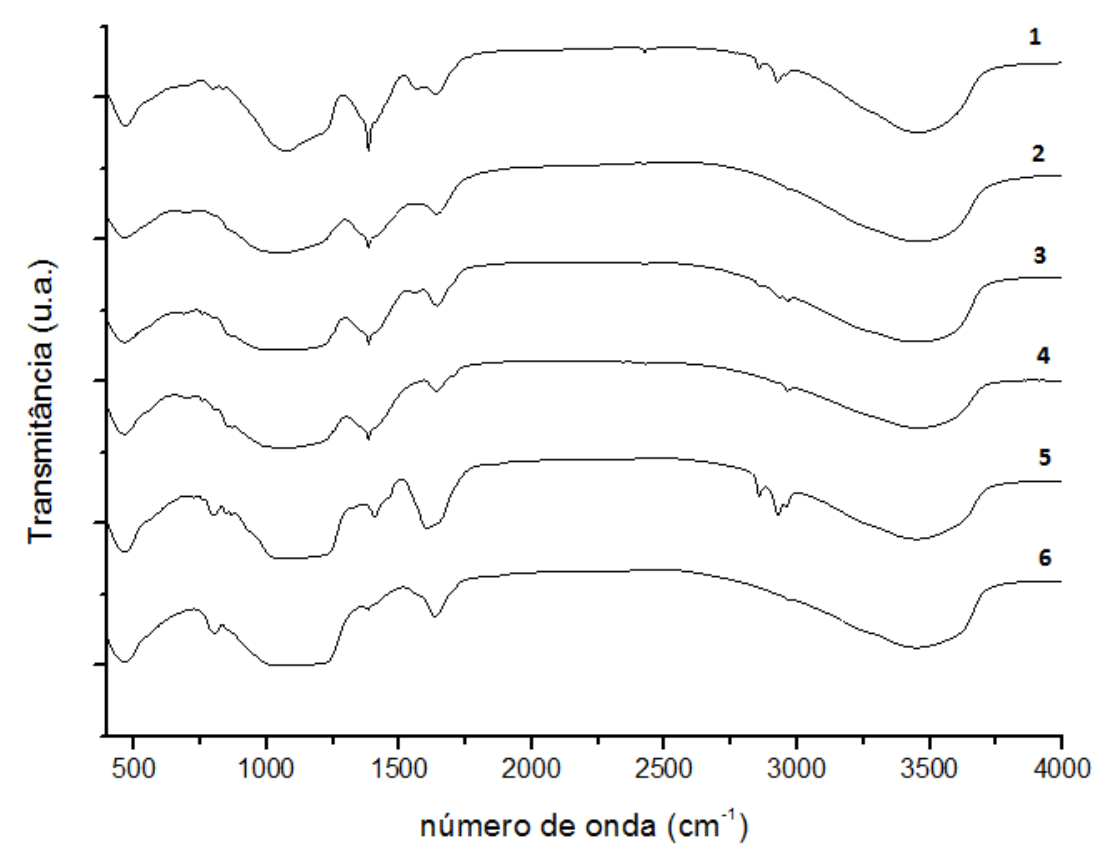

Figura 2 - Espectroscopia na região do infravermelho com transformada de Fourier dos catalisadores após os processos de lavagem.

\section{CONCLUSÕES}

A partir dos resultados obtidos é possível verificar que o poder de complexação do EDTA é maior que o poder de dissolução do etanol na remoção do nitrato de sódio de catalisadores CoNP/SBA-15. A etapa de tratamento térmico, posterior a etapa de lavagem, parece potencializar a remoção dos compostos indesejados. Além disso, é mostrado que o método de preparação utilizado forma catalisadores estáveis em relação à fase metálica, já que aparentemente não houve sinterização das nanopartículas de cobalto (CoNPs) após a etapa de tratamento térmico. Deste modo, a estabilidade térmica da fase metálica (CoNPs) e a eficiência na remoção dos compostos de sódio do catalisador, por meio de tratamento simples de lixiviação, em conjunto com tratamento térmico, permite que os catalisadores apresentem características desejáveis para serem utilizados frente à Síntese de Fischer-Tropsch.

\section{AGRADECIMENTOS}

Os autores agradecem ao CNPq e à CAPES pelo suporte financeiro.

\section{REFERENCIAS}

BALONEK, C. M.; LILLEBØ, A. H.; RANE, S.; RYTTER, E.; SCHMIDT, L. D.; HOLMEN, A. Effect of Alkali Metal Impurities on Co-Re Catalysts for FischerTropsch Synthesis from Biomass-Derived Syngas. Catalysis Letters, v.138, p.8-13, 2010 . 
BEZEMER, G. L.; BITTER, J. H.; KUIPERS, H. P. C. E.; OOSTERBEEK, H.; HOLEWIJN, J. E.; XU, X.; KAPTEIJN, F.; DILLEN, A. J.; JONG, K. P. Cobalt Particle Size Effects in the Fischer-Tropsch Reaction Studied with Carbon Nanofiber Supported Catalysts. Journal of the American Chemical Society, v. 128, n.12, p.3956-3964, 2006.

BORG, Ø.; HAMMER, N.; ENGER, B. C.; MYRSTAD, R.; LINDVÅG, O. A.; ERI, S.; SKAGSETH, T. H.; RYTTER, E. Effect of biomass-derived synthesis gas impurity elements on cobalt Fischer-Tropsch catalyst performance including in situ sulphur and nitrogen addition. Journal of Catalysis, v.279, p.163-173, 2011.

DAVIS, B. H. Fischer-Tropsch synthesis: relationship between iron catalyst composition and process variables. Catalysis Today, v.84, n.1-2, p.83-98, 2003.

DRY, M. E. Practical and theoretical aspects of the catalytic Fischer-Tropsch process. Applied Catalysis A, v.138, n.2, p.319-344, 1996.

LILLEBØ, A. H.; PATANOU, E., YANG, J.; BLEKKAN, E. A.; HOLMEN, A. The effect of alkali and alkaline earth elements on cobalt based Fischer-Tropsch Catalysts. Catalysis Today, v.215, p.60-66, 2013.

MARTÍNEZ, A.; LÓPEZ, F.; MÁRQUEZ, F.; DÍAZ, I. Fischer-Tropsch synthesis of hydrocarbons over mesoporous Co/SBA-15 catalysts: the influence of metal loading, cobalt precursor, and promoters. Journal of Catalysis, v.220, p.486-499, 2003.

O'BRIEN, R. J.; XU, L.; SPICER, R. L.; BAO, S.; MILBURN, D. R.; DAVIS, B. H. Activity and selectivity of precipitated iron Fischer-Tropsch catalysts. Catalysis Today, v.36, n.3, p.325-334, 1997.

RAMOS, A. L. D.; MARQUES, J. J.; SANTOS, V.; FREITAS, L. S.; SANTOS, R. G. V. M.; SOUZA, M. M. V. M. Atual estágio de desenvolvimento da tecnologia GTL e perspectivas para o Brasil. Química Nova, v. 34, n.10, p. 1704-1716, 2011.

TAM, M. S.; GUNTER, G. C.; CRACIUN, R.; MILLER, D. J.; JACKSON, J. E. Reaction and Spectroscopic Studies of Sodium Salt Catalysts for Lactic Acid Conversion. Industrial \& Engineering Chemistry Research, v.35, p.3505-3512, 1997.

WANG, Z.-J.; SKILES, S.; YANG, F.; YAN, Z.; GOODMAN, D. W. Particle size effects in Fischer-Tropsch synthesis by cobalto. Catalysis Today, v.181, n.1, p.75-81, 2012.

YANG, J.; TVETEN, E. Z.; CHEN, D.; HOLMEN, A. Understanding the Effect of Cobalt Particle Size on Fischer-Tropsch Synthesis: Surface Species and Mechanistic Studies by SSITKA and Kinetic Isotope Effect. Langmuir, v.26, n.21, p.16558-16567, 2010.

ZHAO, Y-W.; ZHENG, R. K.; ZHANG, X. X.; XIAO, J. Q. A simple method to prepare uniform Co nanoparticles. IEEE Transactions on Magnetics, v. 39, n.5, p. 2764-2766, 2003.

ZHAO, D.; SUN, J.; LI, Q.; STUCKY, G. D. Morphological control of highly ordered mesoporous silica SBA-15. Chemistry of Materials, v.12, n.2, p.275 - 279, 2000.

ZHAO, D.; FENG, J.; HUO, Q.; MELOSH, N.; FREDRICKSON, G. H.; CHMELKA, B. F.; STUCKY, G. D. Triblock copolymer syntheses of mesoporous silica with periodic 50 to 300 angstrom pores. Science, v.279, n.5350, p.548-552, 1998. 